\title{
Thickness of graphene oxide-based materials as a control parameter
}

\author{
A. Esteban-Arranz ${ }^{1, *}$, M. A. Arranz ${ }^{2}$, M. Morales ${ }^{3}$, R. Martín-Folgar ${ }^{3}$, and J. Álvarez-Rodríguez ${ }^{4, *}$ \\ 1 Dpto. de Ingeniería Química, Universidad de Castilla-La Mancha, Avenida Camilo José Cela, S/N, 13005, \\ Ciudad Real, España; Adrian.Esteban@uclm.es \\ 2 Dpto. de Física Aplicada, Facultad de Ciencias, Av. Camilo José Cela, 10, 13007, Ciudad Real, España; \\ Miguelangel.arranz@uclm.es \\ 3 Grupo de Biología y Toxicología Ambiental, Facultad de Ciencias, UNED, Paseo Senda del Rey, 9, 28040 \\ Madrid, España; mmorales@ccia.uned.es; mfolgar@ccia.uned.es \\ 4 Dpto. de Química Inorgánica y Química Técnica, Facultad de Ciencias, UNED, Paseo Senda del Rey, 9, \\ 28040 Madrid, España; jalvarez@ccia.uned.es \\ * Correspondence: jalvarez@ccia.uned.es; Adrian.Esteban@uclm.es
}

\begin{abstract}
:
Graphene oxide-based materials have been widely used for different applications, such as: biotechnology, electronics, and adsorption or separation technologies amongst other uses. In this study, graphite oxide (GrO), large graphene oxide (lGO) and small graphene oxide (sGO) were synthesized. Monolayer large graphene oxide (mlGO) was detected and isolated in this synthesis prior to IGO separation from GrO. A battery of techniques was applied to elucidate their physicochemical properties. Morphological results acquired by high resolution scanning electron microscopy, transmission electron microscopy and scanning transmission electron microscopy demonstrated the flat and planar structures of these materials. Similar lateral dimensions were found for $1 \mathrm{GO}$ and mlGO unlike sGO. However, based on atomic force microscopy studies, it was able to demonstrate that 1GO presented thicker laminar structures than mlGO. Their crystallography evaluated by $\mathrm{x}$-ray diffraction corroborated the results obtained by the atomic force microscopy studies, since mlGO displayed a diffractogram characteristic of highly exfoliated material. Additionally, Turbiscan experiments revealed a more significant impact from the thickness of these materials in contrast to their lateral dimensions in their colloidal stability properties in aqueous solution. Characterization results were correlated with the optical band gap obtained from the Tauc method of their UV-vis absorption spectra, which could be implemented to characterize in-line the production of these carbon materials to optoelectronic devices.
\end{abstract}

Keywords: 2D materials; monolayer large graphene oxide; small graphene oxide; colloidal stability; thickness.

\section{Introduction}

The exceptional properties of Graphene-based materials (GBM) such as surface area, mechanical and thermal properties amongst others [1], have been exploited for many applications like 2D-membranes [2]; electrical conductors [3], energy [4], biomedicine [5,6], biosensor [7,8], composites [9], optical [10], adsorption [11], batteries [12], and solar cell technologies [13]. Therefore, their production at industrial scale has increased during the last decade [14]. Since A. Geim and K. Novesolov won the Nobel Prize for the isolation of a graphene monolayer via the micromechanical exfoliation of graphite, many production procedures of these materials have been established. Nowadays, there is still an urgent need to define the quality controls for the synthesis of these materials at lab and industrial scale. For the translation of these GBM to more industrial applications, the impact of one or more properties from these materials to a selected process has to 
be further studied. Thus, more detailed routes of synthesis for the control of a specific physicochemical property must be developed.

The editorial board of the Carbon journal [15] suggested a definition for these GBM in relation with some of their physicochemical properties such as their lateral dimensions and thickness. In this work, the oxidized from of graphene, among others, graphene oxide (GO), was defined as " $a$ chemically modified graphene prepared by oxidation and exfoliation that is accompanied by extensive oxidative modification of the basal plane." Based on the fourth principle enumerated in this classification (Base names on crystallography and morphology), the thickness of GBM and the number of layers should be precisely determined. In this way, different graphene oxide- based materials were defined: i) graphite oxide is referred to the bulk material that is produced after the oxidation of graphite. This graphitic material, 3D, can be exfoliated to produce monolayer graphene oxide (ii) or few layers graphene oxide (iii), considered to be 2D materials.

This highly oxidized treatment of the graphite basal plane also produces an increase of its interlayer spacing, due to the incorporation of functional groups and water [16]. These interlayer spaces have acted as active sites for different applications, like adsorption or as a membrane for filtration applications in aqueous solution [17]. Different oxygen species such as carboxylic acids, hydroxyl or epoxide groups are incorporated, providing the GO with a more hydrophilic nature. Therefore, GO has enormous potential to leverage some of the unique properties of graphene for various aspects in future applications.

In this way, GO has attracted much attention for optoelectronics and biotechnology applications due to the ease of tuning its band gap. Previous publications have demonstrated that this band gap could be dependent on the structure of the graphene oxide material. Thus, many experimental approaches have been developed to alter the band gap of GO materials such as: i) chemical modification; ii) thermal exfoliation or iii) photocatalytic reduction among others. It should be noted, a controllable and not-destructive process is needed in order to correctly tune the gap energy for the selected application. M. T. Hasan et al. [18] demonstrated that the band gap of GO could be modified via a controllable ozonation process. They established that the degree of structural changes was time dependent, which could lead to an over oxidation effect of GO and consequently, decreasing GO emissions. Therefore, more controllable synthesis of graphene oxide materials for optoelectronic applications should be investigated.

Recently, a study by R. Ikram et al. on the industrial scalable production of graphene oxide and analytical approaches for synthesis and characterization was published [14]. This work is mainly focused on the optimization and effect of the different reagents and experimental conditions used during the synthesis to the final GO properties. However, the separation process of few layers graphene oxide should be taken into account, since it is found to be mixed with graphite oxide before its purification.

Therefore, the aim of this work was to synthesize, isolate and characterize highly pure graphene oxide-based materials regarding their thickness as a control parameter. Additionally, small graphene oxide was synthesized following F. Rodrigues et al. experimental procedure for comparison purposes [19]. Finally, a more detailed study on their optical properties for future optoelectronic applications was carried out and explained.

\section{Materials and Methods}

Large graphene oxide (1GO) was prepared via Hummer's method following the synthesis published elsewhere [20]. In this procedure, the separation of the graphene oxide layers from the 
graphitic residues was explained and demonstrated. Additionally, F. Rodrigues et al. [19] validated the reproducibility of this experimental procedure and the new synthesis and separation of smaller graphene flakes (sGO). Both research works showed that the solution (Graphite + 1GO) should be thoroughly washed until its $\mathrm{pH}$ value reached 6. However, prior to the solubilization of the 1GO with warm water, in the washing fraction, we observed a very stable orange-yellowish solution that we hypothesized contained large, very thin, and stable graphene oxide layers. Thus, we named it monolayer large graphene oxide (mlGO). Once the 1GO solution was separated from the graphitic material, the latter one was washed several times with warm water to remove the remaining large graphene oxide flakes. Then the graphitic material was recovered and characterized. After that, materials were lyophilized via a freeze-drying process with liquid nitrogen at $77 \mathrm{~K}$ and sublimated (0.004 mbar) until room temperature.

High Resolution Scanning Electron Microscope (HRSEM). Morphological and structural analyses of the different graphene oxide materials were recorded by a GeminiSEM 500 from the ZEISS brand.

Transmission Electron Microscopy (TEM). TEM analyses were carried out on $100 \mu \mathrm{g} \cdot \mathrm{mL}$ ${ }^{1}$ solutions. They were dip-cast on 200 mesh and $3.00 \mathrm{~mm}$ Lacey copper grids and dried at room temperature. Micrographs were acquired by a JEOL 2100, High-Resolution Transmission Electron Microscope (HRTEM) at a voltage of $100 \mathrm{kV}$.

Scanning Transmission Electron Microscope (STEM). Lateral dimensions of the different graphene oxide materials were elucidated by a GeminiSEM 500 from the ZEISS brand in a STEM mode.

Atomic Forced Microscopy (AFM). Graphene Oxide flakes $\left(100 \mu \mathrm{g} . \mathrm{mL}^{-1}\right)$ were deposited on an exfoliated mica coated with $20 \mu \mathrm{L}$ of $0.01 \%$ poly-L-lysine. After washing the excess of material, samples were placed into an oven at $40{ }^{\circ} \mathrm{C}$ to dry overnight. AFM equipment (NT-MDT, Solver) was used in a tapping mode with an AFM tip: high resolution probe (Carbon spike on silicon apex, SHR150, tip radius $<1 \mathrm{~nm})$.

Raman spectroscopy. Spectra between 1000 and $3250 \mathrm{~cm}^{-1}$ were acquired by a Renishaw, InVia spectrometer equipped with a $633 \mathrm{~nm}$ laser set to $1 \%$ power and measured with a 50x objective. All spectra were normalized by the G band intensity with OriginPro 8.5 software.

X-ray diffraction (XRD). Spectra were recorded on a Philips (Panalytical) X'Pert MPD diffractometer. $\mathrm{Cu} \mathrm{K} \alpha 1(1.54056 \AA)$ at $40 \mathrm{kV}$ and $40 \mathrm{~mA}$ was used. A zero background material was used as a sample holder.

Fourier Transform Infrared Spectroscopy (FT-IR). Superficial functional groups of the materials were determined by a Spectrum Two fourier transform infrared (FTIR) spectrometer, from PerkinElmer Inc., with a zinc selenide (ZnSe) crystal. Spectra acquired ranged from 4000 to $450 \mathrm{~cm}^{-1}$, with a $4 \mathrm{~cm}^{-1}$ resolution and 100 scans per sample.

TurbiscanTM Lab Expert stability analyzer. Colloidal stability of the different materials in aqueous solution was established by Turbiscan Stability Index (TSI). Dispersions of graphene oxide in aqueous solution were prepared in a cylindrical vial at $\mathrm{C}_{0}=5 \mu \mathrm{g} \mathrm{mL}-1$ with a total volume of $30 \mathrm{~mL}$. All measurements were carried out without any previous sonication step. 
UV-visible spectroscopy (UV-vis). Optical measurements of graphene oxide solutions were analyzed by UV-vis in a Varian CARY 1 spectrophotometer at room temperature in a 200-800 nm wavelength range.

\section{Results and Discussion}

\subsection{Microscopy}

To determine morphological differences between these materials, HRSEM measurements were carried out. This technique has a lateral resolution of tens of nanometers, which provides greater lateral resolution than optical microscopy. Micrographs acquired are shown in Figure SI.1. GrO presents a multilayer structure with a wrinkled surface due to the oxidation treatment. Otherwise, 1GO, sGO and mlGO display flat and thinner structures than GrO, which are distinctive of graphene-based materials. The lateral dimensions of sGO flakes are noticeably smaller than 1GO and mlGO. The latter seems to present thinner graphene oxide flakes than the other GOs. TEM analyses were carried out to define their 2D structure and lateral dimensions. Micrographs are shown in Figure 1. As it was previously demonstrated by HRSEM, 1GO, sGO and mlGO present characteristic structures of 2D materials. However, GrO shows a wrinkled and thicker structure in comparison to the other materials. It is not possible to establish size distribution profiles of the $1 G O$ and mlGO because of the physical limitations of the microscope. However, it is clear the small lateral dimensions of sGO in comparison to mlGO and $1 G O$ as shown by HRSEM. Additionally, these two last materials seem to present similar lateral dimensions.
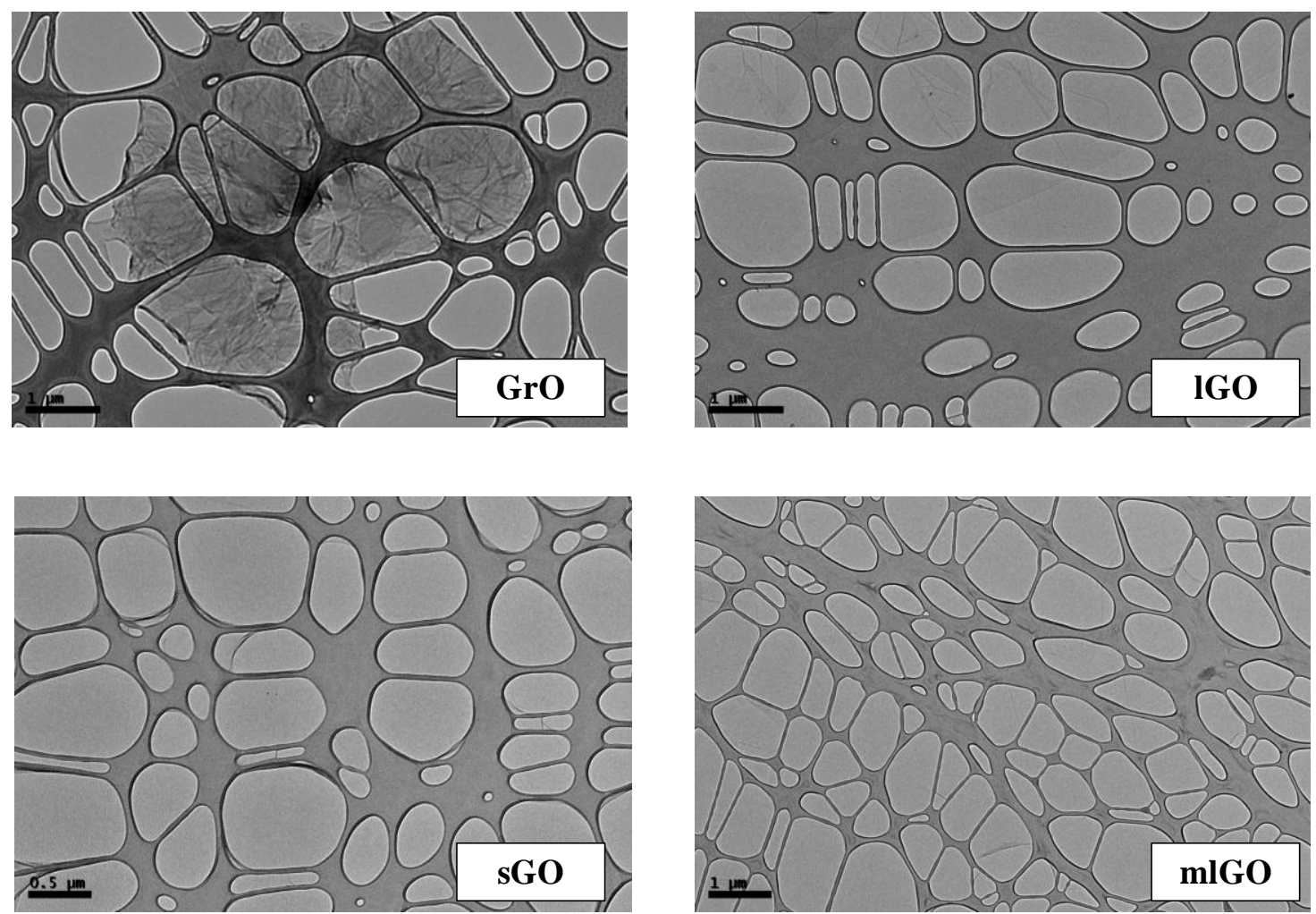

Figure 1. TEM micrographs of GrO, lGO, sGO and mlGO.

STEM analyses were carried out to verify HRSEM and TEM outcomes. Micrographs of 1GO, sGO and mlGO are shown in Figure 2. Smaller graphene oxide flakes of sGO, as predicted in 
HRSEM and TEM analyses, in comparison to $1 \mathrm{GO}$ and mlGO were detected. Additionally, mlGO displays big and well-defined flakes, in the same lateral dimension ranges as 1GO. Therefore, the existence of big and thin flakes is demonstrated in the washing fraction of the material.
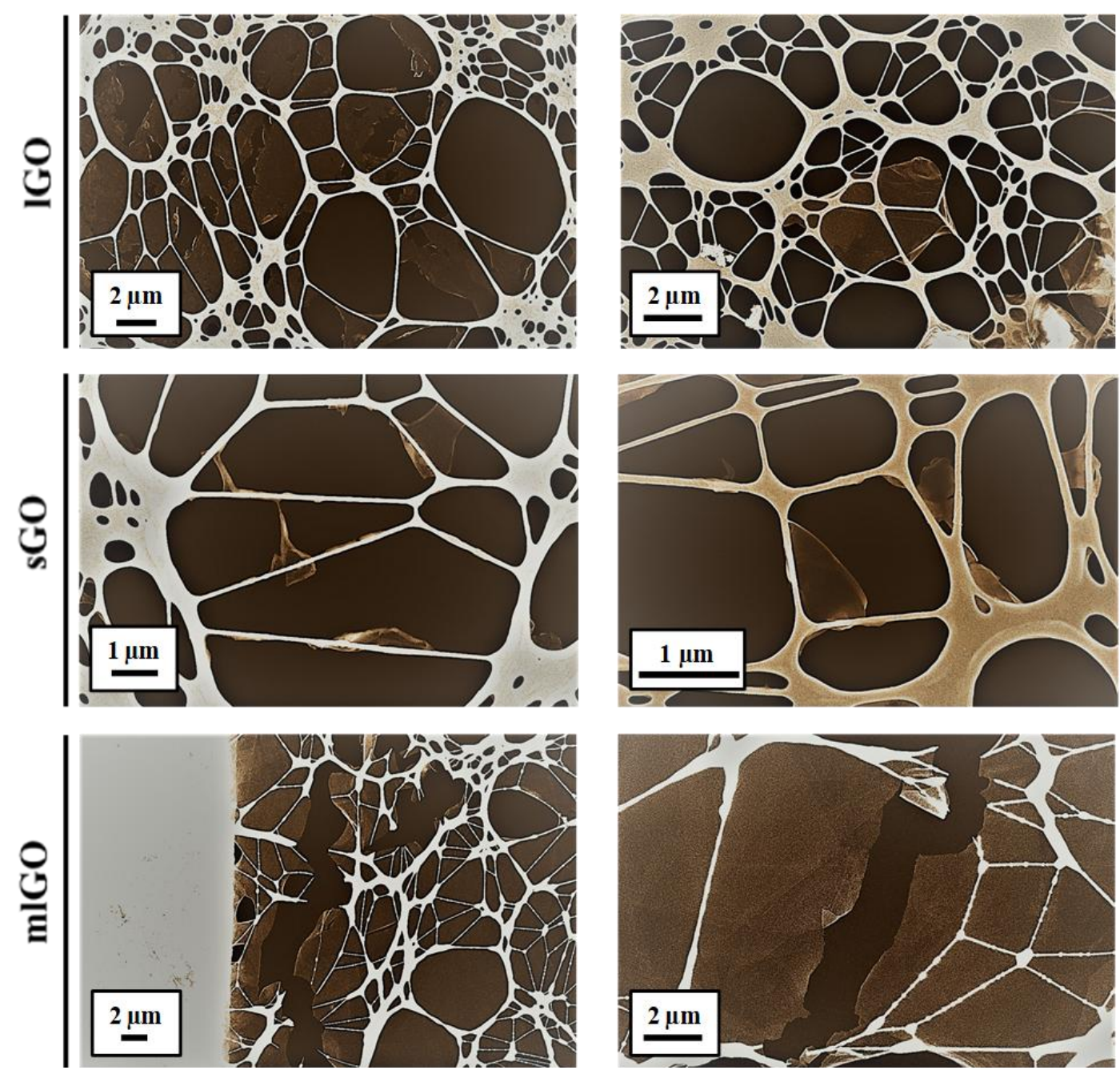

Figure 2. STEM micrographs, colored in brown, of $1 G O$, sGO and mlGO showing their lateral dimensions and structure.

Atomic force microscopy (AFM) is a scanning probe microscopy technique that allows the imaging of the topography of a surface with nanoscale lateral and height resolution. Therefore, lateral dimensions and thickness of a graphene oxide flake can be determined. Figure 3 shows the AFM micrographs of $1 \mathrm{GO}$, sGO and $\mathrm{mlGO}$ and their height profiles. Results show that sGO presents smaller lateral dimensions $(\sim 200-1000 \mathrm{~nm})$ than the rest of the materials, as previously shown in STEM. Big flakes $(6-12 \mu \mathrm{m})$ were found with $1 G O$ and mlGO. However, different thickness values are detected with these last GO materials. Their height profiles reveal that thinner graphene oxide flakes are found with mlGO $(0.62-1 \mathrm{~nm})$ in contrast to $1 G O(1.2-4.5 \mathrm{~nm})$. The thickness of these graphene oxide layers in mlGO corroborates its monolayer structure in distinction to the few layers of graphene oxide $(\mathrm{N} \geq 2)$ detected with $1 G O$. Additionally, sGO presents a comparable thickness (1.1-2.4 nm) to 1GO. These $1 G O$ and sGO results are in accordance with F. Rodriguez values [19]. 

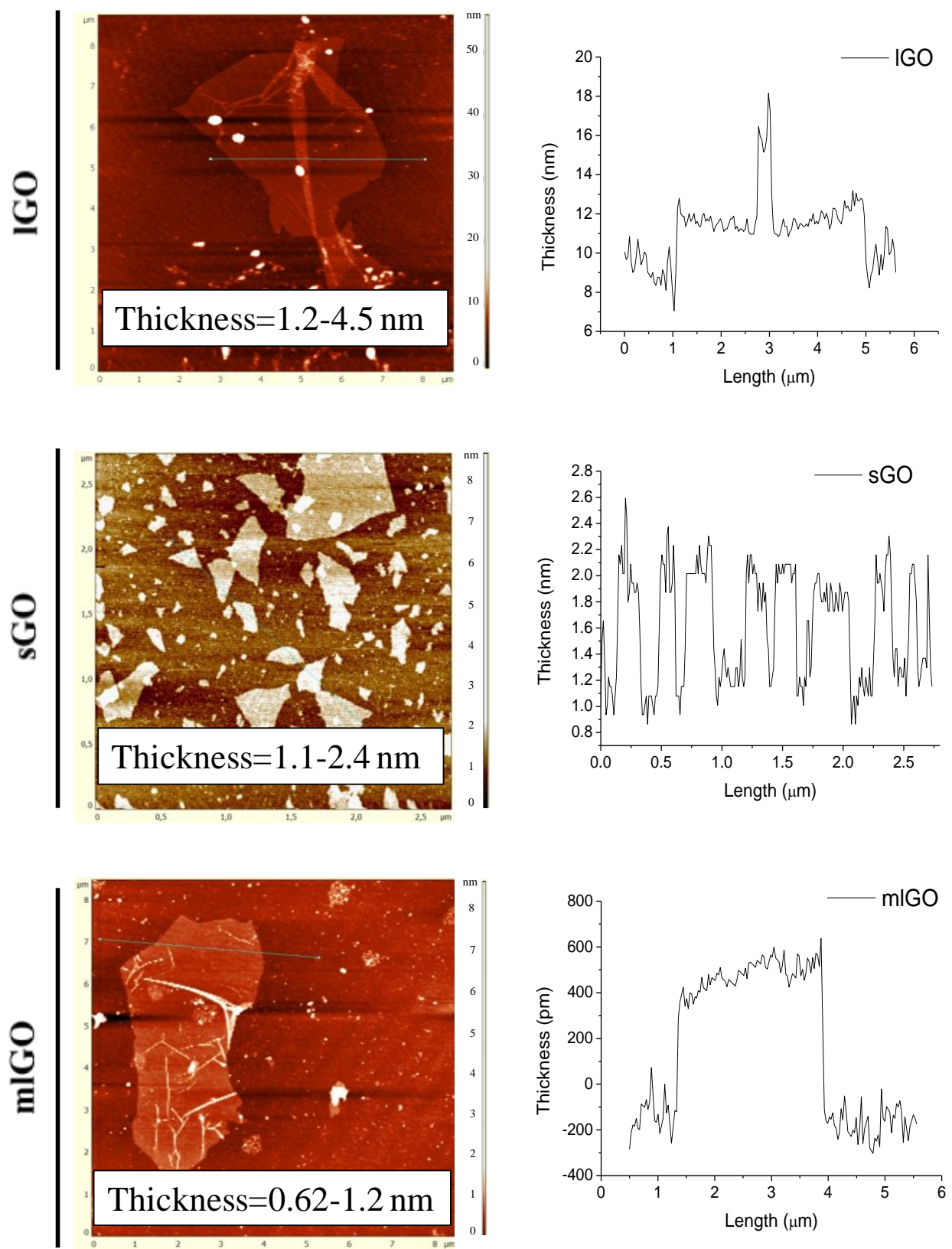

Figure 3. AFM micrographs of $1 \mathrm{GO}$, sGO and mlGO and their respective thickness profiles.

\subsection{Crystallinity}

To determine the structural properties of the resulting GO materials, Raman spectroscopy measurements were carried out. Spectra are depicted in Fig. S1.2. Results show the contribution of two main bands: i) band $\mathrm{D}\left(\sim 1340 \mathrm{~cm}^{-1}\right)$ attributed at the breathing modes of $\mathrm{sp}^{2}$ rings, and ii) $\mathrm{G}$ band located at $\sim 1575 \mathrm{~cm}^{-1}$ for $1 \mathrm{GO}$, sGO and mlGO, which is characteristic of the graphitic domain from the material and $\sim 1582 \mathrm{~cm}^{-1}$ for GrO. Their spectra have been deconvoluted into 5 contributions (D, D', D', $\mathrm{D}^{*}$ and G) following the study carried out by D. López-Díaz et al [21]. The ratio between both bands $\left(\mathrm{ID}_{\mathrm{D}} / \mathrm{IG}_{\mathrm{G}}\right)$ represents the degree of defects from a material [22]. In this case, the values compiled in Table SI.1 shows that all GOs present $\mathrm{ID}_{\mathrm{D}} / \mathrm{I}_{\mathrm{G}}=1.31-1.39$ degree of defects, 
defective graphene, the $\mathrm{D}$ and $\mathrm{D}^{\prime}$ bands are proportional to the type and concentration of defects [23]. As in the case of D. López-Díaz et al. [21] the ID/IG ratio values obtained from our materials are less than 3.5, so consequently, they are ranged in stage 1 or low-defect graphene materials [24]. To define the type of defects found in $\mathrm{GrO}, 1 \mathrm{GO}$, sGO and mlGO, their $\mathrm{AD}^{\prime} / \mathrm{Ag}_{\mathrm{g}} \mathrm{vs}$. $\mathrm{AD} / \mathrm{Ag}$ (Table SI.1) values were plotted [25]. Results are depicted in Figure 4. $\mathrm{An} \mathrm{AD}^{\prime} / \mathrm{AD}_{\mathrm{D}}=0.16$ value was obtained, which is similar to the value presented by A. Eckman et al. for graphene with vacancies as defects (0.14) [26]. These results are in agreement with D. Lopez-Diaz conclusions for GO materials with lateral sizes bigger than $400 \mathrm{~nm}$.

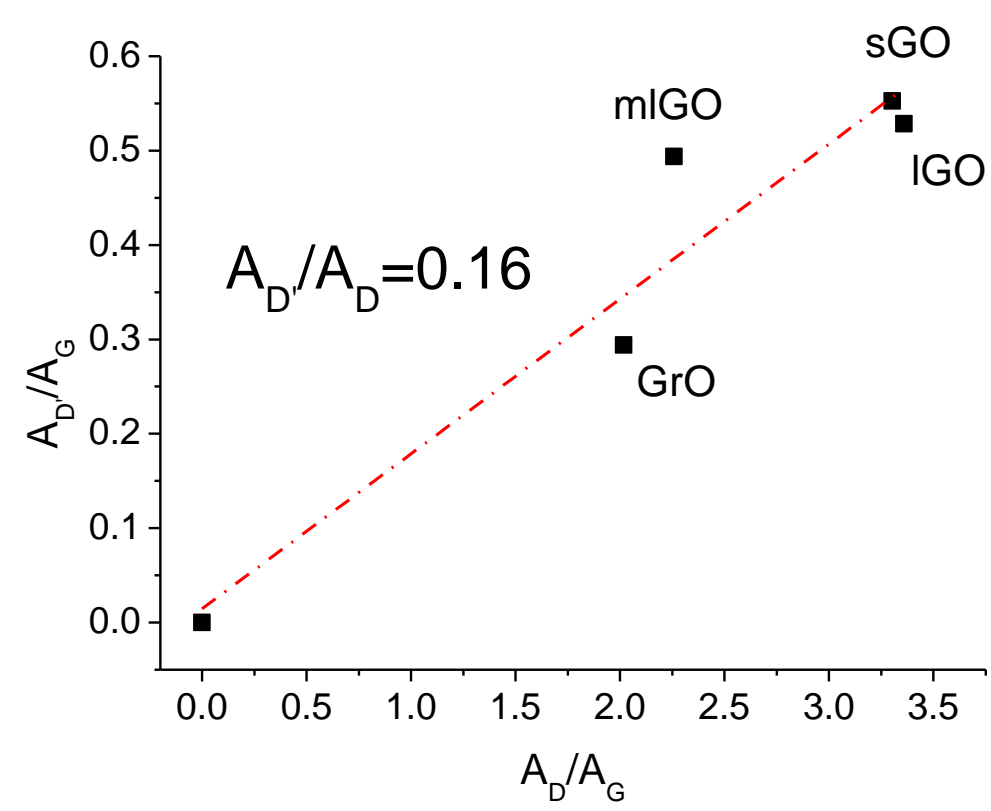

Figure 4. $\mathrm{AD}^{\prime} / \mathrm{Ag}_{\mathrm{g}} \mathrm{vs} . \mathrm{AD} / \mathrm{Ag}_{\mathrm{g}}$ plot to establish the type of defects in these materials. .

X-ray diffraction patterns of the synthesized materials are shown in Figure 5. After the oxidizing treatment, $\mathrm{GrO}$ is formed and consequently, the main peak of the graphite plane (002) is shifted into lower 20. GO materials show a main diffraction peak related to the (001) plane of basal planes at $\mathrm{GrO}\left(2 \theta=12.1^{\circ}\right), 1 \mathrm{GO}\left(2 \theta=11.8^{\circ}\right)$ and $\mathrm{sGO}\left(2 \theta=11.6^{\circ}\right)$, which displacement position is related to the incorporation of oxygen functional groups and water molecules between their layers [27]. It can be observed that GrO presents a more intense and sharper peak than the rest of the GO materials, corroborating its higher graphitizacion degree and the presence of more abundant layers in comparison to the rest of the materials. Similar results were obtained by HRSEM micrographs. The peak of the (002) plane from the starting graphite can only be detected in this GrO material. Previously, AFM experiments demonstrated the difference between the thickness value of IGO and mlGO, despite presenting similar lateral dimensions, as it was also confirmed by HRSEM and STEM micgrographs. These results are in accordance with the XRD outcomes shown in Figure 5, since mlGO diffractogram almost displays a continous line in comparison to $1 G O$ and sGO, characteristic of highly exfoliated graphene oxide materials without crystallinity. 


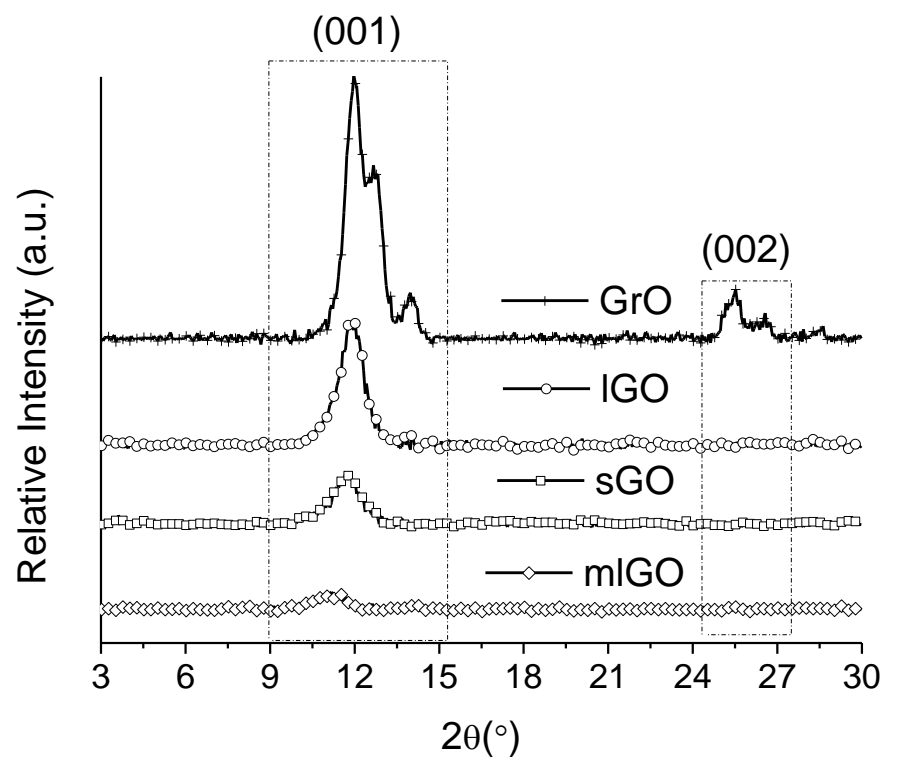

Figure 5. X-ray diffractograms of freeze-dried graphite oxide (GrO), large graphene oxide (lGO), small graphene oxide (sGO) and monolayer large graphene oxide (mlGO).

\subsection{Surface properties}

Surface chemistry of GrO, 1GO, sGO and mIGO was elucidated by FTIR-ATR and is depicted in Figure SI.3. In all cases, similar functional groups are found. A broad band ranged between 3693 and $2913 \mathrm{~cm}^{-1}$ referred to the stretching vibration of hydroxyl groups is detected. This band presents a shoulder characteristic of free hydroxyl radicals. A band centered at $1725 \mathrm{~cm}^{-1}$ assigned to carbonyl groups from carboxylic groups is also identified. A prominent band indicating the existence of hydroxyl and epoxide groups is also detected at $1053 \mathrm{~cm}^{-1}$. Otherwise, bands from the aromatic degree and graphitic carbon are shown at $1400 \mathrm{~cm}^{-1}$ and $1625 \mathrm{~cm}^{-1}$, respectively. This latter band can also be linked with the aromaticity, which is mainly connected to the existence of vicinal hydroxyl groups in acidic surfaces. Similar results were obtained elsewhere [28]. However, even though all materials present the same oxygenated functionalities, some differences have been found. Structural changes of these materials can also be revealed since they have been established as 2D materials. Previously, it was exhibited in Figure SI.1 that GrO presented greater numbers of graphene oxide layers in comparison to the rest of the materials. Consequently, its FT-IR ATR spectrum shows a greater contribution from the aromatic domain and graphitic carbon than the rest of the materials. Otherwise, in the case of $1 G O$ and sGO, both materials show similar spectra, demonstrating the minor differences found between their surface chemistry and structure. Finally, mIGO spectrum shows the least graphitic domain residue, due to its thinner structure, despite having fewer defects than the rest of the materials (Figure SI.2).

\subsection{Colloidal stability}

Colloidal stability of the different GOs materials in aqueous solution was evaluated by Turbiscan experiments. This technique has previously been used to establish the stability of nanoparticles in different solvents [29]. Dai et al. classified the colloidal stability of different graphene oxides in aqueous solution, depending on their Turbiscan Stability Index (TSI) values [30]. A well dispersed and stable graphene oxide would obtain a TSI value lower than five. If this 
TSI value is comprehended between five and twenty, then, sedimentation of GO occurs, but it can be easily re-dispersed. However, if the TSI value obtained is more than twenty, the material is completely precipitated. Colloidal stability results of GrO, 1GO, sGO and mlGO are presented in Figure 6. The TSI of GrO displayed the highest value (14.5) in comparison to 1GO (3.4), sGO (2.1) and mlGO (1.3). This result can be attributed to the characterization outcomes previously obtained, since GrO presented the highest thickness shown by HRSEM and XRD. Otherwise, the rest of the materials obtained TSI values lower than 5, so based on the Dai et al. classification, they can be considered as highly stable materials in aqueous solution. However, some differences between these materials have been found (Figure 5 b). 1GO presented a greater TSI value (3.4) than sGO (2.1), showing that graphene oxide flakes with smaller lateral dimensions display better colloidal stability than bigger ones. Although similar lateral dimensions between mlGO and 1GO are found, the lowest TSI value is acquired with this mlGO material, suggesting that the thickness of the 2D graphene oxide is a more crucial physical parameter than its lateral dimension for colloidal stability applications in aqueous solution.
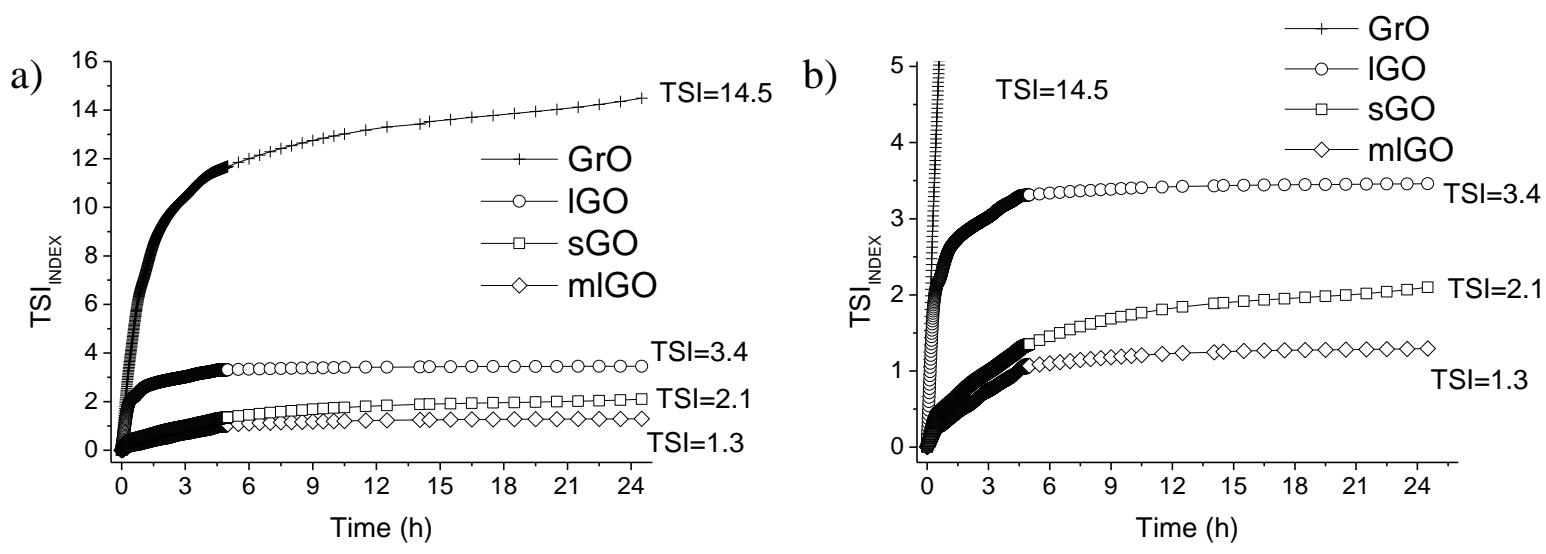

Figure 6. Colloidal stability of $\mathrm{GrO}, 1 \mathrm{GO}$, sGO and mlGO for $24 \mathrm{~h}$.

\subsection{Optical properties}

UV-vis absorption spectra in the wavelength range 200 to $800 \mathrm{~nm}$ of Graphene Oxide dispersions in water are depicted in Figure SI.4. They exhibit two characteristic features: a maximum at $\mathrm{GrO}(226.8 \mathrm{~nm}), 1 \mathrm{GO}(227.0 \mathrm{~nm})$, sGO $(228.3 \mathrm{~nm})$ to $\mathrm{mlGO}(227.7 \mathrm{~nm})$, corresponding to $\pi->\pi^{*}$ transitions of aromatic $C=C$ bonds, and a shoulder at GrO $(298.9 \mathrm{~nm}), 1 \mathrm{GO}(296.6 \mathrm{~nm})$, sGO $(294.2 \mathrm{~nm})$ to $\mathrm{mlGO}(295.7 \mathrm{~nm})$, which can be attributed to $\mathrm{n}->\pi^{*}$ transitions of $\mathrm{C}=\mathrm{O}$ bonds, similar to that reported by Paredes et al.[28].

Analysis of UV-vis absorption spectra by Tauc plots is presented in Figure 7. They let to estimate the optical band gap of these semiconductors. In this method, the tail in the absorption spectrum is characteristic of semiconductor and it is related with the disorder that modifies the energy gap between the valence and conduction bands [31,32]. The energy gap of the samples $\mathrm{GrO}$ $(3.67 \mathrm{eV}), 1 \mathrm{GO}(4.24 \mathrm{eV}), \mathrm{sGO}(4.32 \mathrm{eV})$ to $\mathrm{mlGO}(4.37 \mathrm{eV})$, is enhanced in parallel with the decrease in their size and number of layers. So, bigger lateral size flakes and more stacked basal planes provide a greater band state density, resulting in a modification in the conduction band to a minor value of their energy band gap. These significant band gap differences in graphene oxide materials prove their possible application for optoelectronics and sensor devices [33]. 

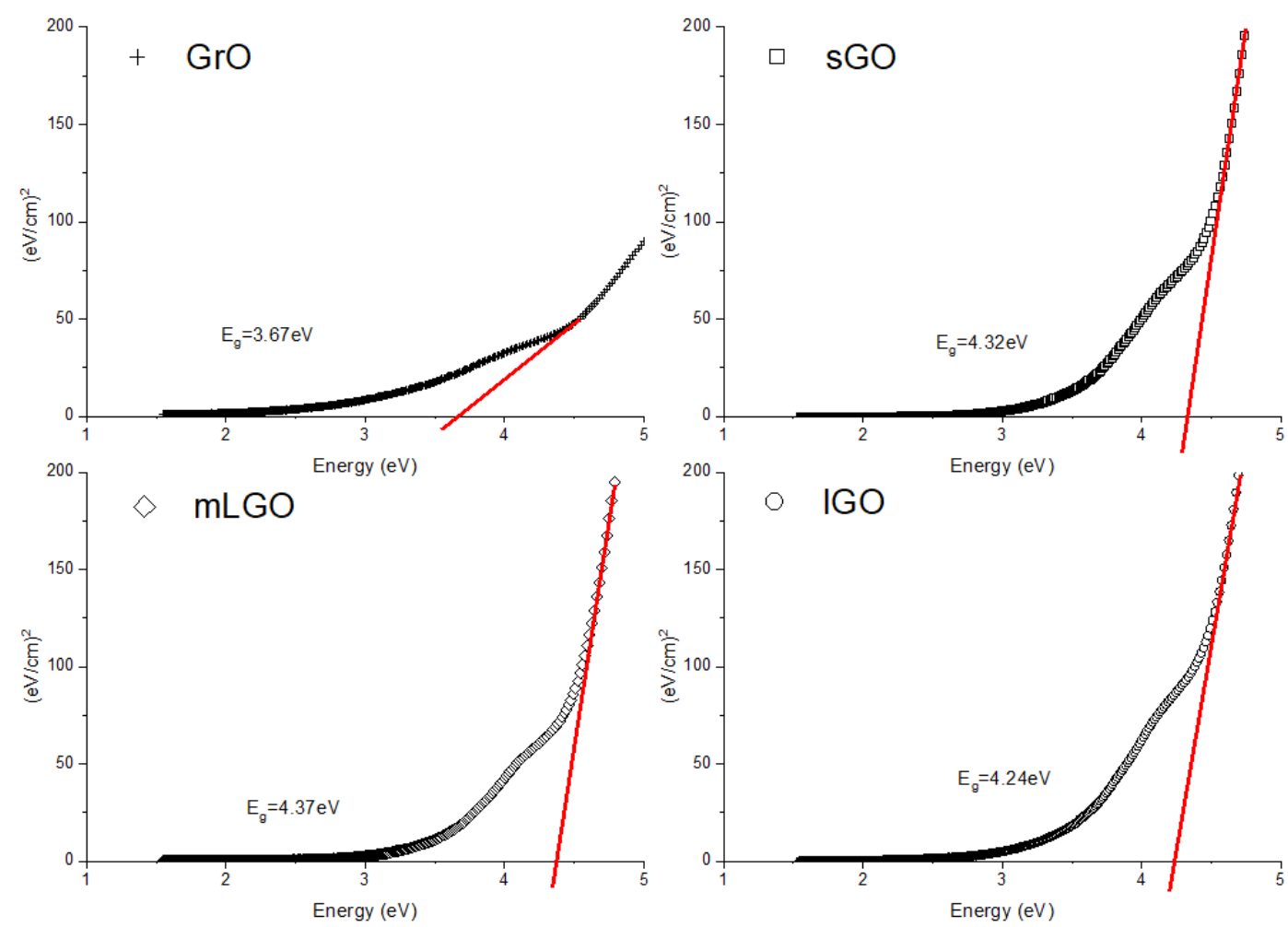

Figure 7. Tauc plots of the GrO (+), sGO $(\square)$, mlGO $(\diamond)$ and $1 \mathrm{GO}(O)$, for the determination of the energy gap.

It is an open discussion in literature the origin of this energy band gap between the electronic states of the oxygenated surface groups $[34,35,36]$ and their surrounded basal planes $[37,38]$. The former is supported by the extension of electronic confinement, density of surface functional groups, and the later to quantum confinement inversely on the size of the basal planes confined by surface functional groups (therefore the ratio surface defects/basal plane size). Our results confirm Hasan et al. [18] studies, in which optoelectronic properties in both, the graphitic size dominium and surface groups, cooperate in the optical band performance. It is suggested an equivalence between the bigger flake size and the higher optical gap value. However, based on our previous solid and liquid characterization results, we recommend that the thickness of the material should be incorporated in this discussion, since it has been demonstrated its critical influence on the band gap energy value. This property could be applied to flexible optoelectronic devices development by the adequate selection of graphene-based material.

\section{Conclusions}

In this study, graphite oxide (GrO), large graphene oxide (lGO) and small graphene oxide (sGO) were synthesized. Additionally, the existence of monolayer large graphene oxide flakes in the washing fraction prior to the solubilization of $1 G O$ with warm water was demonstrated. HRSEM, TEM and STEM techniques showed the planar and flat structures of these 2D materials, where mlGO presented similar lateral dimension as 1 GO. Height profiles obtained by AFM established the thinner structure of mlGO $(0.6-1.2 \mathrm{~nm})$ in comparison to $1 \mathrm{GO}(1.2-4.5 \mathrm{~nm})$ and sGO (1.1-2.4 nm). Vacancies were defined by Raman spectroscopy after the incorporation of oxygen functional groups as the main defects type in these materials. XRD results corroborated the outcomes provided by AFM, since mIGO presented a continous line diffractogram characteristic of 
highly exfoliated material without a crystallinity character. FTIR-ATR spectra showed the same type of oxygen functional groups for all materials, however, more aromatic carbon contribution was detected with $\mathrm{GrO}>1 \mathrm{GO}=\mathrm{sGO}>\mathrm{mlGO}$, being in agreement with our previous evaluation about the number of graphene oxide layers. Additionally, the colloidal stability of these materials in aqueous solution was elucidated by Turbiscan experiments, which revealed the greater impact of their thickness value in contrast to their lateral dimension. Turbiscan stability index results followed the trend: $\mathrm{GrO}(14.5)>\mathrm{IGO}(3.4)>\mathrm{sGO}(2.1)>\operatorname{mlGO}(1.3)$, showing the importance of their thickness parameter for colloidal stability. Based on UV-vis experiments, it was evidenced that the graphite dominium size of GO materials was the main factor of their optical properties. Optical GO band gap in aqueous solution changed in the sequence $\mathrm{mlGO}>\mathrm{sGO}>1 \mathrm{GO}>\mathrm{GrO}$, concluding that large and thinner GO materials promoted greater band gap values.

Results obtained through HRSEM, TEM, STEM, AFM, Raman spectroscopy, XRD, FTIR-ATR, Turbiscan and UV-visible characterization provide critical support in determining GrO, 1GO, sGO and mlGO properties, allowing us to evaluate their impact in future applications with these materials.

Supplementary Materials: Figure SI.1: HRSEM micrographs showing the morphology for the resulting materials, graphite oxide $(\mathrm{GrO})$, large graphene oxide $(\mathrm{GGO})$, small graphene oxide (sGO) and monolayer large graphene oxide (mlGO); Figure SI.2: Raman spectra of graphite oxide (GrO), large graphene oxide (lGO), small graphene oxide (sGO) and monolayer large graphene oxide (mlGO); Figure SI.3: FT-IT ATR results of graphite oxide (GrO), large graphene oxide ( $\mathrm{GO})$, small graphene oxide (sGO) and monolayer large graphene oxide (mlGO), showing their surface chemistry; Figure SI.4: UV-vis absorption spectra of the graphite oxide (GrO, black line), large graphene oxide ( $\mathrm{GO}$, red line), small graphene flakes (sGO, green line) and monolayer large graphene oxide (mlGO, blue line) for the determination of the energy gap and Table SI.1: Raman parameters for graphite oxide (GrO), large graphene oxide (lGO), small graphene oxide (sGO) and monolayer large graphene oxide (mlGO).

Author Contributions: Conceptualization, A.E.A. and J.A.R.; investigation, A.E.A.; AFM investigation, A.E.A and M.A.A. Turbiscan investigation: M.M., R.M.F. and A.E.A; data curation, A.E.A.; writing-original draft preparation, A.E.A. and J.A.R.; writing - review and editing, A.E.A. and J.A.R.; All authors have read and agreed to the published version of the manuscript.

Funding: This research received no external funding.

Conflicts of Interest: The authors declare no conflict of interest.

\section{References}

1. Rao, C.; Sood, A.; Subrahmanyam, K.; Govindaraj, A. Graphene: The New Two-Dimensional Nanomaterial. Angewandte Chemie International Edition, 2009, 48, 7752-7777.

2. Koros, W.J.; Zhang, C. Materials for next-generation molecularly selective synthetic membranes. Nat Mater., 2017, 16, 289-297.

3. Novoselov, K.S.; Geim, A.K.; Morozov, S.V.; Jiang, D.; Zhang, Y.; Dubonos, S. V.; Grigorieva, I. V.; Firsov, A. A. Electric field effect in atomically thin carbon films. Science, 2004, 306, 666-669.

4. Krishnan, D.; Franklin Kim, F.; Luo, J.; Cruz-Silva, R.; Cote, LJ.; Jang, H.D.; Huang, J. Energetic graphene oxide: Challenges and opportunities. Nano Today, 2012, 7, 137-152.

5. Liu, Z.; Robinson, J.T.; Sun, X.M.; Dai, H.J. PEGylated nanographene oxide for delivery of water-insoluble cancer drugs. J. Am. Chem. Soc., 2008, 130, 10876-10877.

6. Barua, S.; Geng, X.; Chen, B. Graphene-based nanomaterials for healthcare applications, Photonanotechnology for Therapeutics and Imaging, 2020; pp. 45-81.

7. Kang XH; Wang J; Wu H; Aksay IA; Liu, J.; Lin, Y.H. Glucose oxidase-grapheme-chitosan modified electrode for direct electrochemistry and glucose sensing. Biosens. Bioelectro., 2009 25,901-905. 
8. Li, Y.; Tang, L.; Deng, D.; He, H.; Yan, X.; Wang, J.; Luo, L. Hetero-structured MnO-Mn33 $\mathrm{O}_{4} @ \mathrm{rGO}$ composites: Synthesis and nonenzymatic detection of $\mathrm{H}_{2} \mathrm{O}_{2}$, Materials Science and Engineering: C, 2021, 118, 111443.

9. Solís Pinargote, N.W.; Smirnov, A.; Peretyagin, N.; Seleznev, A.; Peretyagin, P. Direct Ink Writing Technology (3D Printing) of Graphene-Based Ceramic Nanocomposites: A Review, Nanomaterials, 2020, $10,1300$.

10. Zhai, F.; Feng, Y.; Zhou, K.; Wang, L.; Zheng, Z.; Feng, W. Graphene-based chiral liquid crystal materials for optical applications. J. Mater. Chem. C, 2019, 7, 2146-2171.

11. Ali, I.;Basheer, A.A.; Mbianda, X.Y.;Burakov, A.; Galunin, E.; Burakova, I.; Mkrtchyan, E.; Tkachev, A.; Grachev, V. Graphene based adsorbents for remediation of noxious pollutants from wastewater. Environment International, 2019, 127, 160-180.

12. Patil, K.;Rashidi, S.; Wang, H.; Wei, W. Recent Progress of Graphene-Based Photoelectrode Materials for Dye-Sensitized Solar Cells. International Journal of Photoenergy, 2019, 1-16.

13. Kumar, R.; Sahoo, S.; Joanni, E.; Singh, R.K.; Tan, W.K.; Kar, K.K.; Matsuda, A.Recent progress in the synthesis of graphene and derived materials for next generation electrodes of high performance lithium ion batteries. Progress in Energy and Combustion Science, 2019, 75, 100786.

14. Ikram, R.; Jan, B.M.; Ahmad, W. An overview of industrial scalable production of graphene oxide and analytical approaches for synthesis and characterization. J. Mater. Res. Technol., 2020, 9, 11587-11610.

15. Bianco, A.; Cheng, H.M.; Enoki, T.; Gogotsi, Y.; Hurt, R.H.; Koratkar, N.; Kyotani, T.; Monthioux, M.; Park, C.R.; Tascon, J.M.D.; J. Zhang. J. All in the graphene family - a recommended nomenclature for twodimensional carbon materials Carbon, 2013, 65, 1-6.

16. Esteban-Arranz, A.; Pérez-Cadenas, M.; Muñoz-Andrés, V.; Guerrero-Ruiz, A. Evaluation of graphenic and graphitic materials on the adsorption of Triton X-100 from aqueous solution, Environmental Pollution, 2021, 284, 117161.

17. Hu, M.; Zhenhua Yao, Z.; Xianqin Wang, X. Graphene-Based Nanomaterials for Catalysis. Industrial $\mathcal{E}$ Engineering Chemistry Research, 201756 (13), 3477-3502

18. Hasan, M.; Senger, B.J.; Ryan, C.; Culp, M.; Gonzalez-Rodriguez, R.; Coffer, J. L.; Naumov, A.V. Optical Band Gap Alteration of Graphene Oxide via Ozone Treatment. Sci. Rep., 2017 7, 6411.

19. Rodrigues A.F.; Newman, L.; Lozano, N.; Mukherjee, S.P.; Fadeel, B.; Bussy, C.; Kostarelos, K. A blueprint for the synthesis and characterisation of thin graphene oxide with controlled lateral dimensions for biomedicine. 2D Mater., 2018, 5, 035020.

20. Jasim, D; Lozano, N; Kostarelos, K. Synthesis of few-layered, high-purity graphene oxide sheets from different sources for biology. 2D Mater., 2016, 3, 014006.

21. López-Díaz, D.; López Holgado, M.; García-Fierro, J. L.; Velázquez, M. M. Evolution of the Raman Spectrum with the Chemical Composition of Graphene Oxide; J. Phys. Chem. C, 2017, 121, 20489-20497.

22. Ferrari, A.C.; Meyer, J.C.; Scardaci, V.; Casiraghi, C.; Lazzeri, M.; Mauri, F.; Piscanec, S.; Jiang, D.; Novoselov, K. S.; Roth, S.; Geim, A. K. Raman spectrum of graphene and graphene layers; Phys. Rev. Lett., 2006, 97, 187401.

23. Venezuela, P.; Lazzeri, M.; Mauri, F. Theory of Double-Resonant Raman Spectra in Graphene: Intensity and Line Shape of Defect-Induced and Two-Phonon Bands. Phys. Rev. B: Condens. Matter Mater. Phys. 2011, 84, 035433

24. Ferrari, A C.; Robertson, J. Interpretation of Raman Spectra of Disordered and Amorphous Carbon. Phys. Rev. B. Condens. Matter Mater. Phys., 2000, 61, 14095-14107.

25. Lucchese, M. M.; Stavale, F.; Ferreira, E. H. M.; Vilani, C.; Moutinho, M. V. O.; Capaz, R. B.; Achete, C. A.; Jorio, A. Quantifying Ion-Induced Defects and Raman Relaxation Length in Graphene. Carbon, 2010, 48, 1592-1597.

26. Eckmann, A.; Felten, A.; Mishchenko, A.; Britnell, L.; Krupke, R.; Novoselov, K. S.; Casiraghi, C. Probing the Nature of Defects in Graphene by Raman Spectroscopy. Nano Lett., 2012, 12, 3925-3930.

27. Liu, Z; Duan, X; Zhou, X; Qian, G; Zhou, J; Yuan, W. Controlling and Formation Mechanism of Oxygen Containing Groups on Graphite Oxide; Ind. Eng. Chem. Res. 2014, 53, 253-258.

28. Esteban-Arranz, A.; Compte-Tordesillas, D.; Muñoz-Andrés, V.; Pérez-Cadenas, M.; Guerrero-Ruiz, A. Effect of surface, structural and textural properties of graphenic materials over cooperative and synergetic adsorptions of two chloroaromatic compounds from aqueous solution. Catal. Today, 2018, 301, 104-111. 


\section{1}

29. Esteban-Arranz, A.; de la Osa, A. R.; Garcia-Lorefice, W. E.; Sacristan, J.; Sanchez-Silva, L. Long-Term Performance of Nanomodified Coated Concrete Structures under Hostile Marine Climate Conditions. Nanomaterials, 2021, 11, 869.

30. Dai, J.; Wang, G.; Ma, L.; Wu, C. Study on the surface energies and dispersibility of graphene oxide and its derivatives. J. Mater. Sci., 2015, 50, 3895-3907.

31. Tauc, J.; Grigorovici, R.; Vancu, A. Optical Properties and Electronic Structure of Amorphous Germanium. Physica Status Solidi. (b), 1966, 15, 627-637.

32. O'Learly, S.K.; Lim, P.K. On determining the optical GAP associated with an amorphous semiconductor: A generalization of the tauc model. Solid State Commun., 1997, 104, 17-21.

33. Ojrzynska, M.; Wroblewska, A.; Judek, J.; Malolepszy, A.; Duzynska, A.; Zdrojek, M. Study of optical properties of graphene flakes and its derivatives in aqueous solutions. Optics Express, 2020 28, 7274-7281.

34. Kozawa, D. ; Zhu, X.; Miyauchi, Y; Mouri, S; Ichida, M.; Su,H ; Matsuda, K. Excitonic Photoluminescence from Nanodisc States in Graphene Oxides. The Journal of Physical Chemistry Letters, 2014, 5, 1754-1759.

35. Eda, G.; Lin, YY; Mattevi, C.; Yamaguchi, H.; Chen, HA; Chen, IS; Chen, CW; Chhowalla M. Blue Photoluminescence from Chemically Derived Graphene Oxide. Adv Mater., 2010 22, 505-509.

36. Pal, S. K. Versatile photoluminescence from graphene and its derivatives. Carbon, 2015, 88, 86-112.

37. Galande, C.; Mohite, A.; Naumov, A.; Gao, W.; Ci, L.; Ajayan, A.; Gao, H.; Srivastava, A.; Weisman, R.B.; Ajayan, P.M. Quasi-Molecular Fluorescence from Graphene Oxide. Sci. Rep., 2011, 1, 85.

38. Shang, J.; Ma, L.; Li, J, Ai, W; Yu T.; Gurzadyan G.G. The Origin of Fluorescence from Graphene Oxide. Sci. Rep., 2012, 2. 